\title{
HEALTH EFFECTS OF DIETARY FIBER
}

\author{
Semih Ötles ${ }^{\bowtie}$, Selin Ozgoz \\ Department of Food Engineering, Engineering Faculty, Ege University of Izmir \\ 35100 Bornova Izmir, Turkey
}

\begin{abstract}
Dietary fibre is a group of food components which is resistant to digestive enzymes and found mainly in cereals, fruits and vegetables. Dietary fiber and whole grains contain a unique blend of bioactive components including resistant starches, vitamins, minerals, phytochemicals and antioxidants. Dietary fiber which indigestible in human small intestinal, on the other hand digested completely or partially fermented in the large intestine, is examined in two groups: water-soluble and water insoluble organic compounds. Dietary fiber can be separated into many different fractions. These fractions include arabinoxylan, inulin, pectin, bran, cellulose, $\beta$-glucan and resistant starch. Dietary fibres compose the major component of products with low energy value that have had an increasing importance in recent years. Dietary fibres also have technological and functional properties that can be used in the formulation of foods, as well as numerous beneficial effects on human health. Dietary fibre components organise functions of large intestine and have important physiological effects on glucose, lipid metabolism and mineral bioavailability. Today, dietary fibers are known to be protective effect against certain gastrointestinal diseases, constipation, hemorrhoids, colon cancer, gastroesophageal reflux disease, duodenal ulcer, diverticulitis, obesity, diabetes, stroke, hypertension and cardiovascular diseases. In this review the physicochemical and biological properties of dietary fibers and their important implications on human health will be investigated.
\end{abstract}

Key words: digestion, absorption, obesity, prebiotic, coronary heart disease

\section{INTRODUCTION}

Dietary fiber intake provides many health benefits. A generous intake of dietary fiber reduces risk for developing the diseases including coronary heart disease [Liu et al. 1999], stroke [Steffen et al. 2003], hypertension [Whelton et al. 2005], diabetes [Montonen et al. 2003], obesity [Lairon et al. 2005] and certain gastrointestinal disorders [Petruzziello et al. 2006]. Furthermore, increased consumption of dietary fiber improves serum lipid concentrations [Brown et al. 1999], lowers blood pressure [Keenan et al. 2002], improves blood glucose, control in diabetes [Anderson 2004], promotes regularity [Cummings 2001], aids in weight loss [Birketvedt et al. 2005] and appears to improve immune function [Watzl et al. 2005].

Traditionally, dietary fiber was defined as the portions of plant foods that were resistant to digestion by human digestive enzyme; this included polysaccharides and lignin. More recently, the definition has been expanded to include oligosaccharides, such as inulin, and resistant starches [Jones et al. 2006]. As simple, fibers have been classified as soluble, such as viscous or fermentable fibers (such as pectin) that are fermented in the colon, and insoluble fibers, such as wheat bran, that have bulking action but may only be

『semih.otles@ege.edu.tr 
fermented to a limited extent in the colon [Anderson et al. 2009].

\section{DEFINING DIETARY FIBER}

In the simplest form, carbohydrates can be separated into two basic groups based upon their digestibility in the Gastro Intestinal (GI) tract. The first group (i.e. starch, simple sugars, and fructans) is easily hydrolysed by enzymatic reactions and absorbed in the small intestine. These compounds can be referred to as non-structural carbohydrates, non-fibrous polysaccharides (NFC) or simple carbohydrates. The second group (i.e. cellulose, hemicellulose, lignin, pectin and beta-glucans) are resistant to digestion in the small intestine and require bacterial fermentation located in the large intestine. These compounds can be referred to as complex carbohydrates, non-starch polysaccharide (NSP) or structural carbohydrates and are reflective in Neutral Detergent Fiber (NDF) and Acid Detergent Fiber (ADF) analysis. NSP can be further subdivided into the two general groups of soluble and insoluble. This grouping is based on chemical, physical, and functional properties. Soluble fiber dissolves in water forming viscous gels. They bypass the digestion of the small intestine and are easily fermented by the microflora of the large intestine. They consist of pectins, gums, inulin-type fructans and some hemicelluloses. In the human GI tract, insoluble fibers are not water soluble. They do not form gels due to their water insolubility and fermentation is severally limited. Some examples of insoluble fiber are of lignin, cellulose and some hemicelluloses [James and Mark 2010].

A recent description, as suggested by the American Association of Cereal Chemists, terms dietary fiber as carbohydrate polymers with more than a three degree polymerization which are neither digested nor absorbed in the small intestine. The greater than three degree polymer rule was designed to exclude mono and disaccharides [AACC 2010]. The World Health Organization (WHO) and Food and Agriculture Organization (FAO) agree with the American Association of Cereal Chemists (AACC) definition but with a slight variation. They state that dietary fiber is a polysaccharide with ten or more monomeric units which is not hydrolyzed by endogenous hormones in the small intestine [Codex... 2010]. The known constituents of dietary fiber are listed in Table 1.

Non Starch Polysaccharides (NSP) can be further subdivided into the two general groups of soluble and insoluble. This grouping is based on chemical, physical, and functional properties [Sizer and Whitney 2008]. Soluble fiber dissolves in water forming viscous gels. They bypass the digestion in the small intestine and are easily fermented by the microflora of the large intestine. In the human GI tract, insoluble fibers are not water soluble. They do not form gels due to their water insolubility and fermentation is severally

Table 1. Components of dietary fiber according to the American Association of Cereal Chemists [Jones 2000]

\begin{tabular}{lll}
\hline $\begin{array}{c}\text { Non Starch polysaccharides } \\
\text { and oligosaccharides }\end{array}$ & \multicolumn{1}{c}{ Analagous carbohydrates } & $\begin{array}{c}\text { Lignin substances associated } \\
\text { with the NSP and lignin complex }\end{array}$ \\
\hline Cellulose & $\begin{array}{l}\text { indigestible dextrins } \\
\text { resistant maltodextrins } \\
\text { Hemicellulose }\end{array}$ & waxes \\
Arabinoxylans & synthesized carbohydrates compounds & cutin \\
Arabinogalactans & polydextrose & saponins \\
Polyfructoses & methyl cellulose & suberin \\
Inulin & hydroxypropylmethyl cellulose & tannin \\
Oligofructans & resistant starches & \\
Galacto-oligosaccharides & & \\
Gums, mucilages, pectins & & \\
\hline
\end{tabular}


Table 2. Content in dietary fibre of some seaweeds, fruits, vegetables, legumes and cereals expressed in percentage of dry matter [Escrig and Muniz 2000]

\begin{tabular}{|c|c|c|c|}
\hline & $\begin{array}{l}\text { Insoluble } \\
\text { fibre }\end{array}$ & $\begin{array}{c}\text { Soluble } \\
\text { fibre }\end{array}$ & $\begin{array}{l}\text { Total } \\
\text { fibre }\end{array}$ \\
\hline Nori & 16.8 & 17.9 & 34.7 \\
\hline Hijiki & 16.3 & 32.9 & 49.2 \\
\hline Wakame & 5.3 & 30.0 & 35.3 \\
\hline Ulva lactuta & 16.8 & 21.3 & 38.1 \\
\hline Enteromorpha spp. & 16.2 & 17.2 & 33.4 \\
\hline Himantalia elongata & 7.0 & 25.7 & 32.7 \\
\hline Eisenia byciclis & 14.9 & 59.7 & 74.6 \\
\hline Whole soy & 65.24 & 7.08 & 72.32 \\
\hline Whole wheat & 41.59 & 2.87 & 44.46 \\
\hline Whole corn & 87.47 & 0.40 & 87.87 \\
\hline Rice & 0.75 & 0.19 & 0.94 \\
\hline Beans & 25.64 & 10.85 & 36.49 \\
\hline Brussels sprouts & 30.23 & 6.16 & 36.39 \\
\hline Chickpeas & 16.69 & 1.35 & 18.04 \\
\hline Onions & 13.32 & 3.59 & 16.89 \\
\hline Potatoes & 4.85 & 2.14 & 6.99 \\
\hline Apricots & 44.92 & 26.43 & 71.35 \\
\hline Peaches & 39.53 & 27.30 & 66.83 \\
\hline Apples & 55.57 & 18.56 & 74.13 \\
\hline
\end{tabular}

limited. Most fiber containing foods include approximately one-third soluble and two-third insoluble fiber [Wong and Jenkins 2007].

Current recommendations for dietary fiber intake are related to age, gender, and energy intake, and the general recommendation for adequate intake (AI) is $14 \mathrm{~g} / 1000 \mathrm{kcal}$ [USDA 2005]. This AI includes nonstarch polysaccharides, analogous carbohydrates (e.g., resistant starches), lignin, and associated substances [Witwer 2008]. Using the energy guideline of 2000 $\mathrm{kcal} /$ day for women and $2600 \mathrm{kcal} /$ day for men, the recommended daily dietary fiber intake is $28 \mathrm{~g}$ /day for adult women and $36 \mathrm{~g} /$ day for adult men.

\section{HEALTH BENEFITS OF DIETARY FIBER}

\section{Cardiovascular}

Cardiovascular diseases, including coronary heart disease (CHD), stroke, and hypertension, affect more than 80 million people and are the leading causes of morbidity and mortality in the United States. In 2005, CHD was the leading cause of death and strokes were the third leading cause of death in the United States [American... 2008]. While CHD is the most prevalent cause of death, it is probably the most modifiable; an estimated $82 \%$ of CHD is attributed to lifestyle practices such as diet, physical activity, and cigarette abuse [Stampfer et al. 2000] and 60\% is attributed to dietary patterns [Kris-Etherton et al. 2002].

High levels of dietary fiber intake are associated with meaningfully lower prevalence rates for coroner heart disease, stroke, and peripheral vascular disease [Merchant et al. 2003]; major risk factors, such as hypertension, diabetes, obesity, and dyslipidemia, are also less common in individuals with the highest levels of fiber consumption [Lairon et al. 2005].

The impact of dietary fiber or whole grain consumption on the prevalence of these conditions is summarized in Table 3. In the analyses of prospective cohort studies, the observed protective effect of dietary fiber intake was very similar to the effects of whole grains but "fellow travelers" with fiber, such as magnesium, other minerals, vitamins, and antioxidants, may have significant complementary beneficial effects [Anderson 2004, Anderson et al. 2007].

Seven cohort studies presenting observations for over 158,000 individuals indicate that CHD disease prevalence is significantly lower (29\%) in individuals with the highest intake of dietary fiber compared to those with the lowest intake (Table 3). Specifically, the relative risk, computed by variance weighting (fixedeffect metaanalysis) is 0.71 for individuals in the highest quintile for dietary fiber intake compared to those in the lowest quintile [Anderson et al. 2009].

In the United States, CVD affects approximately one-third of the adult US population and CHD is the leading cause of death. Higher intakes of dietary fiber compared to lower consumption levels are associated with significantly lower rates of CVD and lower prevalence of CVD risk factors. Persons with the highest levels of fiber consumption have a $29 \%$ lower risk 
Table 3. Dietary fiber intake related to relative risk for disease based on estimates from prospective cohort studies [Anderson et al. 2009]

\begin{tabular}{lccc}
\hline \multicolumn{1}{c}{ Disease } & $\begin{array}{c}\text { Number of } \\
\text { subjects } \\
\text { (studies) }\end{array}$ & $\begin{array}{c}\text { Relative } \\
\text { risk* }\end{array}$ & $95 \%$ CI \\
\hline $\begin{array}{l}\text { Coronary heart } \\
\text { disease }\end{array}$ & $158,327(7)$ & 0.71 & $0.47-0.95$ \\
Stroke** & $134,787(4)$ & 0.74 & $0.63-0.86$ \\
Diabetes & $239,485(5)$ & 0.81 & $0.70-0.93$ \\
Obesity & $115,789(4)$ & 0.70 & $0.62-0.78$ \\
\hline
\end{tabular}

*Relative risks adjusted for demographic, dietary, and nondietary factors.

**Estimates related to whole-grain consumption, total dietary fiber and cereal fiber.

for CHD than those with the lowest intakes. Soluble fiber intake of about $6 \mathrm{~g} /$ day is accompanied by reductions in serum LDL-cholesterol values of around 5.4\% and estimated risk for CHD of about $9 \%$. Increased fiber intake modestly lowers the blood pressure of the general population but is accompanied by reductions of systolic and diastolic blood pressure, respectively, of $-6 \mathrm{mmHg}$ and $-4 \mathrm{mmHg}$ for hypertensive individuals. Higher fiber intakes are associated with improved measures for body weight, visceral adiposity, insulin sensitivity, and inflammatory markers. Moderate increases in fiber intake, especially soluble fiber, are likely to have significant favourable effects on risk and progression of CVD [Anderson et al. 2009].

Many studies support the inverse relationship of dietary fiber and risk for CHD. However, more recent studies found interesting data illustrating that for every $10 \mathrm{~g}$ of additional fiber added to a diet the mortality risk of CHD decreased by $17-35 \%$. Risk factors for CHD include hypercholesterolemia, hypertension, obesity and type two diabetes. It is speculated that the control and treatment of these risk factors underlie the mechanisms behind DF and CHD prevention. First, soluble fibers have been shown to increase the rate of bile excretion therefore reducing serum total and LDL cholesterol. Second, short chain fatty acid production, specifically propionate, has been shown to inhibit cholesterol synthesis. Third, dietary fiber demonstrates the ability to regulate energy intake thus enhancing weight loss or maintenance of a healthier body weight. Fourth, either through glycemic control or reduced energy intake, dietary fiber has been shown to lower the risk for type two diabetes. Fifth, DF has been shown to decrease pro-inflammatory cytokines such as interleukin-18 which may have an effect on plaque stability. Sixth, increasing DF intake has been shown to decrease circulating levels of C-Reactive protein (CRP), a marker of inflammation and a predictor for CHD [James and Mark 2010].

In a long term clinical study, Jensen et al. [2004] reported that an increased daily consumption of bran significantly decreased the risk of coronary heart disease in healthy adult men. This is most likely due to the data reported by Qureshi et al. [2002] who found that $10 \mathrm{~g}$ of rice bran consumed for eight weeks was able to decrease serum total cholesterol, LDL cholesterol and triglycerides. The mechanisms behind these effects may be twofold. The reduction in cholesterol levels is likely due to an increase in bile acid synthesis. Andersson et al. [2002] found that oat bran doubled the serum concentration of $7 \alpha$-hydroxy-4-cholesten-3-one $(\alpha-\mathrm{HC})$, which is a metabolite in the synthesis of bile acids that is oxidized from $7 \alpha$-hydroxycholesterol. The reduction in serum triglyceride levels may be a result of a decreased absorption of fat from the small intestine.

Theuwissen and Mensink [2008] found that, many well-controlled intervention studies have shown that four major water-soluble fiber types $\beta$-glucan, psyllium, pectin and guar gum effectively lower serum LDL cholesterol concentrations, without affecting HDL cholesterol or triacylglycerol concentrations. Furthermore, epidemiological studies suggest that a diet high in water-soluble fiber protects against CVD. These findings underlie current dietary recommendations to further increase water-soluble fiber intake.

\section{Obesity}

Nearly $66 \%$ of U.S. adults are overweight or obese resulting in an increased risk of health problems including; diabetes, CVD, and certain types of cancer. Although there are multiple factors that could contribute to obesity, the primary cause is due to an increase in the energy absorption, energy expenditure ratio. Therefore, limiting energy absorption is critical when treating obesity. Scientists have taken this a step 
further and studied the effect of other dietary aspects that may serve in weight regulation, including dietary fiber. Increasing dietary fiber consumption may decrease energy absorption by way of diluting a diet's energy availability while maintaining other important nutrients [Lattimer and Haub 2010].

Substantial research has been conducted to evaluate the effect of dietary fiber and body weight, most of all which show an inverse relationship between dietary fiber intake and change in body weight. Tucker and Thomas [2009] supported this statement in a study consisting of 252 middle aged women. They observed that over a 20 month period participants lost an average of $4.4 \mathrm{lbs}$ due to an $8 \mathrm{~g}$ increase in dietary fiber per $1000 \mathrm{kcal}$. This weight loss was primarily due to decreased body fat. It should be recognized that the correlation between dietary fiber and weight change was independent of many other potential factors including age, baseline fiber and fat intakes, activity level, and baseline energy intake.

Koh-Banerjee et al. [2004] concur with the above findings and also suggest a dose-response relationship. They reported that for every $40 \mathrm{~g} / \mathrm{d}$ increase in whole grain intake, weight gain decreased by $1.1 \mathrm{lbs}$. Moreover, bran seemed to play an important role in the reduction of weight gain by $0.8 \mathrm{lbs}$ per $20 \mathrm{~g} / \mathrm{d}$ intake.

Dietary fiber's ability to decrease body weight or attenuate weight gain could be contributed to several factors. First, soluble fiber, when fermented in the large intestine, produces glucagon-like peptide (GLP-1) and peptide YY (PYY). These two gut hormones play a role inducing satiety. Second, dietary fiber may significantly decrease energy intake [Tucker and Thomas 2009]. Women who consumed increased levels of fiber tended to also have a decreased consumption of dietary fat. Third, dietary fiber may decrease a diet metabolizable energy (ME), which is gross energy minus the energy lost in the feces, urine and combustible gases. Baer et al. [1997] observed that an increased consumption of dietary fiber resulted in a decrease in the ME of the diet. This may be attributed to the fact that fat digestibility decreased as dietary fiber increased. Also, as dietary fiber intake increases, the intake of simple carbohydrates tends to decrease. Although, dietary fiber still contributes to the total caloric content of a diet, it is much more resistant to digestion by the small intestine and even somewhat resistant in the large intestine.
Both soluble and insoluble fiber may lead to weight loss. However, there seems to be a relationship between the type of diet (high or low fat) and the type of fiber consumed. Insoluble fiber may play a more important role for weight loss during consumption of a high fat diet. Since resistant starch is a constituent of dietary fiber and undergoes the same digestion as insoluble fiber, comparing resistant starch and insoluble fiber may give us a better understanding of how dietary fiber can be used to treat and prevent obesity. Adding resistant starch to a diet dilutes its ME, but not to the degree of insoluble fiber. Numerous studies have found the same inverse relationship between dietary fiber and weight gain [Koh- Banerjee et al. 2004, Du et al. 2010].

\section{Diabetes}

Type two diabetes has increased exponentially over the past several years. Since 1990, self reported diabetes increased by $61 \%$ [Mokdad et al. 2003]. Although other risk factors such as obesity, lack of physical activity and smoking are precursors for the disease, dietary factors also seem to play a significant role. Type two diabetes results from decreased insulin sensitivity and hyperglycemia. For that reason, a primary dietary factor of particular concern is carbohydrate intake [Lattimer and Haub 2010].

Meyer et al. [2000] observed that total carbohydrates had no effect on the risk of diabetes. However, the type of carbohydrate (non structural carbohydrates and dietary fiber) was significantly related. Therefore, it is important to understand a foods glycemic index or load. Glycemic index ranks total carbohydrate intake according to their immediate postprandial glucose response in comparison to a reference group such as glucose or white bread. Carbohydrates with a low glycemic index result in a smaller glucose/insulin response. Simple small chain carbohydrates would be considered to have a higher glycemic index since it produces higher blood glucose concentrations.

$\mathrm{Hu}$ et al. [2001] found that excess body fat was the single most important determinant of type two diabetes, poor nutrition was also a large influential factor. Moreover, women consuming a poor diet significantly increased their risk of developing type two diabetes. Poor diet was classified as a diet high in saturated fat, low dietary fiber and high non structural 
carbohydrates. This diet would be consistent with a high glycemic load being higher in easily digestible and rapidly absorbable carbohydrates [Hu et al. 2001]. In a supportive, long term (eight year) study of over 90,000 female nurses, Schulze et al. [2004] found a positive correlation between glycemic index and risk of type two diabetes. This association was significant even after adjusting for age, body mass index (BMI) and family history. Several means have been proposed to understand the physiology behind the relationship of glycemic index and diabetes. First, carbohydrates with a higher glycemic index produce higher blood glucose levels. This chronic hyperglycemia is suggested to lead to the dysfunction of beta cells in the pancreas thus decreasing insulin release. Second, due to an over abundance of energy (i.e., high glycemic load) tissues such as skeletal muscle, liver and adipose become resistant to insulin. It is important to note that the inverse relationship between dietary fiber and diabetes observed by Meyer et al. [2000] and Schulze et al. [2004] was independent of age and body weight. $\mathrm{Hu}$ et al. [2001] supported these findings while correcting for age, fat intake, smoking, alcohol, family history, exercise, and body weight. Therefore, it seems that dietary fiber is associated with type two diabetes, independent of other compounding factors.

Meyer et al. [2000] using healthy middle aged women, observed a strong $(P=0.0012)$ inverse relationship between insoluble fiber and the risk of type two diabetes while soluble fiber had no effect. Montonen et al. [2003] also found the same results in healthy middle aged men and women consuming increased levels of whole rye bread. Interestingly, fiber from fruits and vegetables had no effect on the risk of developing type two diabetes. Earlier studies have agreed with these findings. A large epidemiological study of 42,000 men found that dietary fiber from fruits or vegetables had no effect on the risk of diabetes. However, dietary fiber from whole cereal grains showed a significant decrease in diabetes occurrence. Daily intakes of fiber among all groups were similar [Salmeron et al. 1997].

Weickert and Pfeifer [2008] found that an increased intake of cereal fiber significantly improved whole body glucose disposal resulting in an $8 \%$ improvement of insulin sensitivity. This suggests that the mechanisms behind insoluble fiber are more peripheral and not limited to nutrient absorption. First, an accelerated secretion of glucose-dependent insulintropic polypeptide (GIP) was observed directly after the ingestion of an insoluble fiber in healthy women. GIP is an incretin hormone which stimulates postprandial insulin release. Second, insoluble fiber can result in a reduced appetite and food intake [Samra and Anderson 2007] and this may lead to a decreased caloric intake and BMI. Third, short chain fatty acids, via fermentation, have been shown to reduce postprandrial glucose response [Ostman et al. 2002]. Early research demonstrated that lipid infusions impaired glucose utilization and oral acetate could decrease free fatty acids (FFA) in the blood. According to Kelley and Mandarino [2000] increases in FFA in the blood can inhibit glucose metabolism through the inhibition of GLUT 4 transporters. Therefore, short chain fatty acids, by way of decreasing serum free fatty acids, may reduce blood glucose levels through competition in insulinsensitive tissues.

The inverse relationship between cereal grains and diabetes may also be attributed to an increased consumption of magnesium. Increased intake of magnesium has been shown to decrease the incidence of type two diabetes [Meyer et al. 2000]. Hypomagnesemia is common among diabetics and has been associated with a reduction of tyrosine kinase at the insulin receptor. This may impair the action of insulin thus leading to insulin resistance [Paolisso and Barbagallo 1997].

\section{Gastrointestinal}

Dietary fibers affect the entire gastrointestinal tract from the mouth to the anus. High-fiber foods usually have lower energy density and take longer to eat [Haber et al. 1977]. Soluble fibers usually delay gastric emptying. Soluble fibers may act to slow transit of food materials through the small intestine while insoluble fibers tend to create "intestinal hurry" [Cummings 2001]. In the small intestine, dietary fibers can reveal responses of a wide variety of gastrointestinal hormones that serve as incretins to stimulate insulin release and affect appetite [Anderson 2008]. Some fibers bind bile acids and impede micelle formation, thus increasing fecal excretion of bile acids and cholesterol [Kirby et al. 1981]. In the colon, fermentable fibers increase bacterial mass with some acting as prebiotics to promote health-promoting bacteria such as 
Lactobacilli and Bifidobacteria [Roberfroid 2005]. Insoluble fibers are especially effective in increasing fecal mass and promoting regularity [Cummings 2001].

Recently Drzikova et al. [2005] found that the in vitro binding of bile acids increased with increasing proportions of oat bran, total and insoluble dietary fibre, as well as b-glucan, in pre-digested oat-based extrudates. The results of the study show, that the source, the chemical composition and the pre-treatment of dietary fibre preparations strongly influence their ability to interact with bile acids.

The consumption of b-glucan and resistant starchrich diets results in several beneficial physiological effects. Thus, b-glucan lowers the postprandial blood glucose and insulin responses in normal individual. Serum cholesterol and lipoprotein concentrations can be reduced in humans and animals by b-glucans from oat and barley or by cereal products. A reason for the cholesterol-lowering effects of b-glucan-rich diets may be the greater excretion of bile acids [Drzikova et al. 2005].

Early observers suggested that dietary fiber intake decreased the prevalence of hiatal hernias and gastroesophageal reflux disease (GERD), peptic ulcer disease, gallbladder disease, appendicitis, diverticular disease, colorectal cancer, and hemorrhoids [Burkitt and Trowell 1975]. Rigorous evaluation of the prevalence of these diseases suggests that high levels of dietary fiber intake, compared to low levels, may be associated with a decreased prevalence of the following conditions: esophageal cancer [Wu et al. 2007], GERD [El-Serag et al. 2005], gastric cancer [Wu et al. 2007], peptic ulcer disease [Aldoori et al. 1997], gallbladder disease [Tsai et al. 2004], diverticular disease [Aldoori et al. 1997], constipation and hemorrhoids [Cummings 2001].

Limited data suggest that individuals with higher fiber intakes have a lower prevalence of GERD [El-Serag et al. 2005]. Guar gum and, possibly, other soluble fibers are associated with low levels of gastric acid production, which may protect from GERD and duodenal ulcer disease. The available evidence strongly indicates that high fiber intakes are associated with lower prevalence of duodenal ulcer disease. The available evidence strongly indicates that high fiber intakes are associated with lower prevalence of duodenal ulcer disease than lower fiber intakes, and the epidemiological evidence is moderately well supported by clinical trials [Ryan-Harshman and Aldoori 2004].

Irritable bowel syndrome is one of the most common gastrointestinal functional disorders worldwide. It is a complex disorder and includes the following symptoms: abdominal pain or discomfort, bloating, and diarrhea and/or constipation. While wheat bran often increases symptoms other fiber supplements such as methylcellulose, partially hydrolysed guar gum, and psyllium have been reported to alleviate symptoms [Anderson et al. 2009]. In clinical trials, reductions in symptoms in groups receiving placebo, as well as those receiving fiber are usually dramatic and statistically significant, thus confounding the assessment of the therapeutic intervention. The sympathetic support of the primary care provider combined with highly selective therapeutic interventions with dietary fiber in foods or supplements can often be very effective in reducing symptoms [Giannini et al. 2006]. Judicious use of soluble fibers may offer benefits for persons with ulcerative colitis in remission [Seidner et al. 2005]. Diverticular disease is the fifth most common gastrointestinal condition in Western countries and is one of the classical fiber-deficiency diseases. A generous intake of dietary fiber is considered to be protective, ameliorative, and preventive of recurrences [Frieri et al. 2006]. Increased intake of dietary fiber is commonly used for the prevention and management of constipation or hemorrhoids. Wheat bran, high-fiber cereals, and fiber supplements are widely used by consumers, which represents common knowledge of their beneficial effects [Anderson et al. 2009].

Inulin has demonstrated the ability to contribute to the health of the human large intestine as a prebiotic. They demonstrated that inulin stimulated the growth of Bifidobacteria while restricting the growth of potential pathogenic bacteria such as E. coli, Salmonella, and Listeria. This could prove to be beneficial in such disorders as ulcerative colitis and C. difficile infections. Rafter et al. [2007] agreed with these findings and suggested they were the underlying mechanisms behind the observation that inulin decreased biological compounds associated with colonic cancer, including reduced colorectal cell proliferation and water induced necrosis, decreased exposure to genotoxins, and decreased interleukin-2 release. 
Several recent clinical studies showed that oral pectin supplementation to children and infants reduced acute intestinal infections and significantly slowed diarrhea. This is thought to be due to a reduction in pathogenic bacteria such as Shigella, Salmonella, Klebsiella, Enterobacter, Proteus and Citrobacter. This is supported by Olano-Martin et al. [2002] who observed that pectin stimulated the growth of certain strains of Bifidobacteria and Lactobacillus in vitro. These bacteria are considered to be directly related to the health of the large intestine and their concentrations depict a healthy microflora population.

Kahlon et al. [2001] found that high-fiber diets fed to rats resulted in significantly greater wet and dry fecal weights than low-fiber diets. Bran diets resulted in significantly higher fecal moisture than cellulose diets. Cecum lengths increased significantly with bran diets compared with cellulose diets. The wheat bran diet resulted in significantly higher stomach weights than with cellulose diets. Stomachs were heavier and cecal lengths were greater with bran diets than with cellulose diets; however, a high-cellulose diet resulted in increased colon weight. Except for higher fiber digestibility of coarse bran, bran particle size had no significant effects. Healthful effects of wheat bran may be associated with gastrointestinal morphology and function. Fecal bulking and decreased intestinal transit time can prevent constipation and may dilute or reduce absorption of toxic or carcinogenic metabolites, thus improving gastrointestinal health and lowering the risk of tumor development and cancer.

Ferguson and Harris [1996] found that two groups of mechanisms have been proposed for the way dietary fibres may protect against colorectal cancer: those in which the dietary fibre may act directly and those in which the dietary fibre may have an indirect effect as a consequence of it being degraded by colonic bacterial enzymes and the products fermented.

\section{Cancer}

Cancer continues to be one of the number one health concerns of populations worldwide. Most cancers strike both men and women at about the same rate, with exception of cancers of the reproductive system. Of particular concern is cancer of the colon, ranking among the top 3 forms of cancer in the U.S.A., for both men and women. Colon cancer is also one of the leading causes of cancer morbidity and mortality among both men and women in the Western countries, including the U.S.A. Historical observational and epidemiological studies from around the world have long supported that increased consumption of fruits and vegetables and high fiber intake provide a protective relationship between dietary fiber intake and colon cancer incidence. Early results from a European investigation, the European Prospective Investigation of Cancer (EPIC), involving more than half a million people in 10 European countries, indicates that dietary fiber provides strong protective effects against colon and rectal cancers. Poorly fermented fiber, such as that in cereal brans, has direct effects in the colon by promoting laxation, decreasing transit time, and binding substances such as bile acids and carcinogens. However, evidence to date is insufficient to determine if decreased colon cancer risk is a beneficial effect of this type of fiber. Of particular interest is the utilization of fermentable fiber by the colonic microbiota that can result in changes to the numbers and types of bacteria and, more importantly, changes to their metabolic activities in terms of the formation of genotoxins, carcinogens, and tumor promoters. Selective prebiotic fiber sources, such as inulin, resistant starches, and some oligosaccharides, act as selective substrate for bacteria that produce specific short-chain fatty acids (SCFA) and can lower the intestinal $\mathrm{pH}$. The SCFA butyrate has been shown to increase apoptosis in human colonic tumor cell lines. Apoptosis is a mechanism where excess or redundant cells are removed during development and restricted tissue size is maintained. The apoptosis process is thus an innate cellular defense against carcinogenesis. Evidence suggests that increasing the numbers of Bifidobacteria in the colon and reducing intestinal $\mathrm{pH}$ has a direct impact on carcinogenesis in the large intestine. Possible mechanisms for the anticarcinogenic and antitumorigenic effect of highly fermentable fibers are not completely understood and require further research. However, it is likely that some or all are involved in a metabolic chain reaction for the inhibitory effect to ocur. The primary mechanisms involved with these effects are proposed to be: a reduction in the production of carcinogenic substances by decreasing the amount of pathogenic bacteria in the colon [Rumney and Rowland 1995]; and/or lowering the colonic $\mathrm{pH}$ to affect $\mathrm{pH}$-dependent enzymatic 
reactions; for example, secondary bile acid formation [Rowland et al. 1998]; and/or reducing the amount of carcinogenic substances available to colonic mucosa by adsorption of the substances to the cell wall of the microbiota, by speeding up the intestinal transit time and by increasing colonic contents and thus diluting all components; and/or exerting inhibiting effects on initiation and promotionstages in colon cancer formation in which SCFA, particularly butyric acid, may play a key role [Tungland and Meyer 2006].

\section{Immune system}

Inulin and other oligofructoses have been the most extensively studied dietary fibers. They act, to stimulate growth of bifidobacteria in the colon. Bifidobacteria and lactobacilli are healthpromoting bacteria that generate short-chain fatty acids and stimulate the immune system. Fairly extensive animal studies have documented the favourable effect of inulin on the immune system and preliminary studies in humans support the hypotheses generated from animal studies. Inulin and oligofructoses are not digested by pancreatic enzymes thus, they enter the colon virtually intact. In the colon, inulin is fermented completely by the microbacteria and it promotes the growth of bifidobacteria. Short-chain fatty acids (SCFA) result from this fermentation process. Other fibers are also fermented to generate SCFA, but the bifidobacteria effects of non-oligofructose fibers are not as well characterised. The proposed health benefits of bifidobacteria include the following:

- protection from intestinal infection

- lowering of intestinal $\mathrm{pH}$ for formation of acids after assimilation of carbohydrates

- reduction of the number of potentially harmful bacteria; production of vitamins and antioxidants

- activation of intestinal function and assistance in digestion and absorption, especially of calcium

- bulking activity to prevent and treat constipation

- stimulation of the immune response

- potential reduction in the risk for colorectal cancer. Limited studies in humans have indicated that inulin supplementation increases the fecal bacterial contents of bifidobacteria and has favourable effects on the types and amounts of circulating lymphocytes. Inulin and oligofructoses have been studied most extensively, but favourable effects of fermentable soluble fibers have been demonstrated for oat b-glucan, gumArabic, and others.

The role of prebiotic fibers in infant nutrition and health, especially for non-breastfed infants, is generating a great deal of interest. The studies suggest that supplementation with a prebiotic fiber mixture has the following benefits: promotes postnatal immune development, decreases respiratory infections and atopic dermatitis and improves bowel function.

The therapeutic potential for using prebiotic fibers in the treatment of inflammatory bowel disease is being examined. In animal studies, prebiotic fibers have reduced gut inflammation in a number of animal experimental models. Early studies also indicate that prebiotic fibers significantly reduce the risk of infection in liver transplant patients. Preliminary human studies have shown favourable responses for individuals with ulcerative colitis, Crohn's disease, or pouchitis [Anderson et al. 2009].

\section{Mineral bioavailability}

Certain fiber sources from fruits and vegetables that have cation exchange capacity from unmethylated galacturonic acid residues and phytic acid from cereal fibers, have been found to depress the absorption and retention of several minerals. However, certain highly fermentable fibers have resulted in improved metabolic absorption of certain minerals, such as calcium, magnesium, and iron, even when phytic acid is present at lower concentrations. These compounds include pectin, various gums, resistant starches, cellulose, certain oligosaccharides like soy and fructooligosaccharides, inulin, lactulose, and related sugars. Mineral absorption has generally been accepted as stemming from diffusion in the small intestine. However, studies now indicate that highly fermentable fibers, such as inulin and fructooligosaccharides, also promote mineral absorption in the colon. Through their fermentation by colonic microbiota and subsequent SCFA production, these fiber components stimulate the proliferation of epithelial cells in the cecocolon and reduce the luminal $\mathrm{pH}$. Al salts, especially calcium, magnesium, and iron, in the luminal content and increase their diffusive absorption via the paracellular route. In particular, the accumulation of calcium phosphate in the large intestine and the solubilization of minerals by SCFA are likely to play an essential role in the enhanced mineral 
absorption in the colon. Also, a recent study has demonstrated that fructooligosaccharides (inulin) stimulate the transcellular route of calcium absorption in the large intestine, as indicated by increased concentrations of calbindin-D9k, a calcium binding protein that plays an important role in intestinal calcium transport [Tungland and Meyer 2006].

\section{CONCLUSIONS}

In a simplified definition, dietary fiber is a carbohydrate that resists digestion and absorption and may or may not undergo microbial fermentation in the large intestine. This definition is essentially the basis to its correlation between consumption levels and possible health benefits. Dietary fiber consists of many different constituents, however; some are of particular interest and include arabinoxylan, inulin, $\beta$-glucan, pectin, bran and resistant starches. These individual components of dietary fiber have been shown to significantly play an important role in improving human health. Current research is paying particular attention to these elements although further research is needed to better understand particular health claims and the mechanisms involved. A high level of fiber intake has health-protective effects and disease-reversal benefits. Persons who consume generous amounts of dietary fiber, compared to those who have minimal fiber intake, are at lower risk for developing; Cardiovascular health disease, hypertension, diabetes, obesity, and certain gastrointestinal diseases. Increasing the intake of high fiber foods or fiber supplements improves serum lipoprotein values, lowers blood pressure, improves blood glucose control for diabetic individuals, aids weight loss, and improves regularity.

\section{REFERENCES}

AACC. 2010. Adopts oat bran definition. [online], http:// www.aaccnet.org/news/pdfs/ OatBran.pdf [accessed: 12 October 2010].

Aldoori W.H., Giovannucci E.L., Stampfer M.J., Rimm E.B., Wing A.L., Willett W.C., 1997. Prospective study of diet and the risk of duodenal ulcer in men. Am. J. Epidemiol. 145, 42-50.

American Heart Association. 2008. Cardiovascular disease statistics. [online], www.americanheart.org/presenter. jhtml?identifier $=4478$ [accessed: 6 May 2008].
Anderson J.W., 2004. Whole grains and coronary heart disease: the whole kernel of truth. Am. J. Clin Nutr. Rev. 80, 1459-1460.

Anderson J.W., 2008. Dietary fiber and associated phytochemicals in prevention and reversal of diabetes. In: Nutraceuticals, glycemic health and type 2 diabetes. Eds V.K. Pasupuleti, J.W. Anderson. Blackwell Publ. Profes. Ames, Iowa, 111-142.

Anderson J.W., Baird P., Davis R.H., Ferreri S., Knudtson M., Koraym A., Valerie Waters W., Williams C.L., 2009. Health benefits of dietary fiber. Nutr. Rev. 67 (4), 188-205.

Anderson J.W., Conley S.B., 2007. Whole grains and diabetes. In: Whole grains and health. Eds L. Marquart, D.R. Jr. Jacobs, G.H. McIntosh, K. Poutanen, M. Reicks. Blackwell Publ. Profes. Ames, Iowa, 29-45.

Andersson M., Ellegard L., Andersson H., 2002. Oat bran stimulates bile acid synthesis within $8 \mathrm{~h}$ as measured by 7 alpha-hydroxy-4-cholesten-3-one. Am. J. Clin. Nutr. 76, 1111-1116.

Baer D.J., Rumpler W.V., Miles C.W., Fahey G.C., 1997. Dietary fiber decreases the metabolizable energy content and nutrient digestibility of mixed diets fed to humans. J. Nutri. Rev. 127, 579-586.

Birketvedt G.S., Shimshi M., Erling T., Florholmen J., 2005. Experiences with three different fiber supplements in weight reduction. Med. Sci. Monit. 11, 15-18.

Brown L., Rosner B., Willett W.W., Sacks F.M., 1999. Cholesterol lowering effects of dietary fiber: a meta-analysis. Am. J. Clin. Nutri. Rev. 69, 30-42.

Burkitt D.P., Trowell H.C., 1975. Refined carbohydrate foods and diseases: Some implications of dietary fibre. Academic Press, London.

Codex Alimentarius Commission. 2010. [online], http:// www.codexalimentarius. net/download/standards/34/CXG _002e.pdf [accessed: 12 October 2010].

Cummings J.H., 2001. The effect of dietary fiber on fecal weight and composition. In: CRC handbook of dietary fiber in human nutrition. Ed. G.A. Spiller. CRC Press, Boca Raton, FL, 183-252.

Drzikova B., Dongowski G., Gebhardt E., Habel A., 2005. The composition of dietary fibre-rich extrudates from oat affects bile acid binding and fermentation in vitro. Food Chem. 90, 181-192.

Du H.D., Daphne van der A., Boshuizen H.C., Forouhi N.G., Wareham N.J., Halkjaer J., Tjonneland A., Overvad K., Jakobsen M.U., Boeing H., 2010. Dietary fiber and subsequent changes in body weight and waist circumference in European men and women. Am. J. Clin. Nutr. Rev. 91, 329-336. 
El-Serag H.B., Satia J.A., Rabeneck L., 2005. Dietary intake and the risk of gastro-oesophageal reflux disease: a cross sectional study in volunteers. Gut 54 (1), 11-17.

Escrig A.J., Muniz F.J.S., 2000. Dietary fibre from edible seaweeds: chemical structure, physicochemical properties and effects on cholesterol metabolism. Nutr. Rev. 20, 585- 598.

Ferguson L.R., Harris P.J., 1996. Studies on the role of specific dietary fibres in protection against colorectal cancer. Mutat. Res. 350, 173-184.

Frieri G., Pimpo M.T., Scarpignato C., 2006. Management of colonic diverticular disease. Digestion 73, 58-66.

Giannini E.G., Mansi C., Dulbecco P., Savarino V., 2006. Role of partially hydrolyzed guar gum in the treatment of irritable bowel syndrome. Nutr. Rev. 22, 334-342.

Haber G.B., Heaton K.W., Murphy D., Burroughs L.F., 1977. Depletion and disruption of dietary fibre. Effects on satiety, plasmglucose, and serum-insulin. Lancet 2, 679-682.

Hu F.B., Manson J.E., Stampfer M.J., Colditz G., Liu S., Solomon C.G., Willett W.C., 2001. Diet, lifestyle, and the risk of type 2 diabetes mellitus in women. N. Engl. J. Med. 345, 790-797.

James M.L., Mark D.H., 2010. Effects of dietary fiber and its components on metabolic health. Nutrients 2 (12), 1266-1289.

Jensen M.K., Koh-Banerjee P., Hu F.B., Franz M., Sampson L., Gronbaek M., Rimm E.B., 2004. Intakes of whole grains, bran, and germ and the risk of coronary heart disease in men. Am. J. Clin. Nutri. Rev. 80, 1492-1499.

Jones J., 2000. Update on defining dietary fiber. Cereal Foods World 45, 219-220.

Jones J.R., Lineback D.M., Levine M.J., 2006. Dietary reference intakes: implications for fiber labeling and consumption: a summary of the International Life Sciences Institute North American FiberWorkshop, June 1-2. Washington, DC. Nutri. Rev. 64, 31-38.

Kahlon T.S., Chow F.I., Hoefer J.L., Betschart A.A., 2001. Effect of wheat bran fiber and bran particle size on fat and fiber digestibility and gastrointestinal tract measurements in the Rat. Cereal Chem. 78 (4), 481-484.

Keenan J.M., Pins J.J., Frazel C., Moran A., Turnquist L., 2002. Oat ingestion reduces systolic and diastolic blood pressure in patients with mild or borderline hypertension: a pilot trial. J. Fam. Pract. 51, 369-375.

Kelley D.E., Mandarino L.J., 2000. Fuel selection in human skeletal muscle in insulin resistance - A reexamination. Diabetes 49, 677-683.

Kirby R.W., Anderson J.W., Sieling B., 1981. Oat-bran intake selectively lowers serum low-density lipoprotein cholesterol concentrations of hypercholesterolemic men. Am. J. Clin. Nutri. Rev. 34, 824-829.
Koh-Banerjee P., Franz M.V., Sampson L., Liu S.M., Jacobs D.R., Spiegelman D., Willett W., Rimm E., 2004. Changes in whole-grain, bran, and cereal fiber consumption in relation to 8-y weight gain among men. Am. J. Clin. Nutri. Rev. 80, 1237-1245.

Kris-Etherton P.M., Etherton T.D., Carlson J., Gardner C., 2002. Recent discoveries in inclusive food-based approaches and dietary patterns for reduction in risk for cardiovascular disease. Curr. Opin. Lipidol. 13, 397-407.

Lairon D., Arnault N., Bertrais S., 2005. Dietary fiber intake and risk factors for cardiovascular disease in French adults. Am. J. Clin. Nutri. Rev. 82, 1185-1194.

Lattimer J.M., Haub M.D., 2010. Effects of dietary fiber and its components on metabolic health. Nutrients 2, 1266-1289.

Liu S., Stampfer M.J., Hu F.B., 1999. Whole-grain consumption and risk of coronary heart disease: results from the Nurses' Health study. Am. J. Clin. Nutri. Rev. 70, 412-419.

Merchant A.T., Hu F.B., Spiegelman D., Willett W.C., Rimm E.B., Ascherio A., 2003. Dietary fiber reduces peripheral arterial disease risk in men. J. Nutri. Rev. 133, 3658-3663.

Meyer K.A., Kushi L.H., Jacobs D.R., Slavin J., Sellers T.A., Folsom A.R., 2000. Carbohydrates, dietary fiber, and incident type 2 diabetes in older women. Am. J. Clin. Nutri. Rev. 71, 921-930.

Mokdad A.H., Ford E.S., Bowman B.A., Dietz W.H., Vinicor F., Bales, V.S., Marks J.S., 2003. Prevalence of obesity, diabetes, and obesity-related health risk factors. J. Am. Med. Assoc. 289, 76-79.

Montonen J., Knekt P., Jarvinen R., Aromaa A., Reunanen A., 2003. Whole-grain and fiber intake and the incidence of type 2 diabetes. Am. J. Clin. Nutri. Rev. 77, 622-629.

Olano-Martin E., Gibson G.R., Rastell R.A., 2002. Comparison of the in vitro bifidogenic properties of pectins and pectic-oligosaccharides. J. Appl. Microbiol. 93, 505-511.

Ostman E.M., Liljeberg-Elmstahl H.G., Bjorck I.M., 2002. Barley bread containing lactic acid improves glucose tolerance at a subsequent meal in healthy men and women. J. Nutri. Rev. 132, 1173-1175.

Paolisso G., Barbagallo M., 1997. Hypertension, diabetes mellitus, and insulin resistance: The role of intracellular magnesium. Am. J. Hypertens. 10, 346-355.

Petruzziello L., Iacopini F., Bulajic M., Shah S., Costamagna G., 2006. Review article: uncomplicated diverticular disease of the colon. Aliment. Pharmacol. Ther. 23, 1379-1391.

Qureshi A.A., Sami S.A., Khan F.A., 2002. Effects of stabilized rice bran, its soluble and fiber fractions on blood 
glucose levels and serum lipid parameters in humans with diabetes mellitus Types I and II. J. Nutri. Biochem. 13, 175-187.

Rafter J., Bennett M., Caderni G., Clune Y., Hughes R., Karlsson P.C., Klinder A., O'Riordan M., O'Sullivan G.C., Pool-Zobel B., Rechkemmer G., Roller M., Rowland I., Salvadori M., Thijs H., Van Loo J., Watzl B., Collins J.K., 2007. Dietary synbiotics reduce cancer risk factors in polypectomized and colon cancer patients. Am. J. Clin. Nutri. Rev. 85, 488-496.

Roberfroid M.B., 2005. Introducing inulin-type fructans. Br. J. Nutri. Rev. 93, 13-25.

Rowland I.R., Rumney C.J., Coutts J.T., Lievense L.C., 1998. Effect of Bifidobacterium longum and inulin on gut bacterial metabolism and carcinogen-induced aberrant crypt foci in rats. Carcinogenesis 19, 281-285.

Rumney C., Rowland I.R., 1995. Nondigestible oligosaccharides-potential anti-cancer agents. BNF Nutri. Bull. 20, 194-203.

Ryan-Harshman M., Aldoori W., 2004. How diet and lifestyle affect duodenal ulcers. Review of the evidence. Can. Fam. Physician. 50, 727-732.

Salmeron J., Ascherio A., Rimm E.B., Colditz G.A., Spiegelman D., Jenkins D.J., Stampfer M.J., Wing A.L., Willett W.C., 1997. Dietary fiber, glycemic load, and risk of NIDDM in men. Diabetes Care 20, 545-550.

Samra R., Anderson G.H., 2007. Insoluble cereal fiber reduces appetite and short-term food intake and glycemic response to food consumed 75 min later by healthy men. Am. J. Clin. Nutr. Rev. 86, 972-979.

Seidner D.L., Lashner B.A., Brzezinski A., 2005. An oral supplement enriched with fish oil, soluble fiber, and antioxidants for corticosteroid sparing in ulcerative colitis: a randomized, controlled trial. Clin. Gastroent. Hepatol. 3, 358-369.

Schulze M.B., Liu S., Rimm E.B., Manson J.E., Willett W.C., Hu F.B., 2004. Glycemic index, glycemic load, and dietary fiber intake and incidence of type 2 diabetes in younger and middle-aged women. Am. J. Clin. Nutr. Rev. 80, 348-356.

Sizer F., Whitney E., 2008. Nutrition: Concepts and controversies. Thomson Wadsworth, Belmont, CA, USA.

Stampfer M.J., Hu F.B., Manson J.E., Rimm E.B., Willett W.C., 2000. Primary prevention of coronary heart disease in women through diet and lifestyle. New Eng. J. Med. 343, 16-22.

Steffen L.M., Jacobs D.R., Stevens J., Shahar E., Carithers T., Folsom A.R., 2003. Associations of whole-grain, refined grain, and fruit and vegetable consumption with risks of all-cause mortality and incident coronary artery disease and ischemic stroke: the Atherosclerosis Risk in Communities (ARIC) Study. Am. J. Clin. Nutr. Rev. 78, 383-390.

Theuwissen E., Mensink R.P., 2008. Water-soluble dietary fibers and cardiovascular disease. Phys. Behav. 94, 285-292.

Tsai C.J., Leitzmann M.F., Willett W.C., Giovannucci E.L., 2004. Longterm intake of dietary fiber and decreased risk of cholecystectomy in women. Am. J. Gastroenterol. 99, 1364-1370.

Tucker L.A., Thomas K.S., 2009. Increasing total fiber intake reduces risk of weight and fat gains in women. J. Nutr. Rev. 139, 576-581.

Tungland B.C., Meyer D., 2006. Nondigestible oligo- and polysaccharides (dietary fiber): Their physiology and role in human health and food. Compr. Rev. Food Sci. Food Saf. 20, 97-98.

Watzl B., Girrbach S., Roller M., 2005. Inulin, oligofructose and immunomodulation. Br. J. Nutr. Rev. 93, 49-55.

Weickert M.O., Pfeiffer A.F.H., 2008. Metabolic effects of dietary and prevention of diabetes. J. Nutr. Rev. 138, 439-442.

Whelton S.P., Hyre A.D., Pedersen B., Yi Y., Whelton P.K., He J., 2005. Effect of dietary fiber intake on blood pressure: a metaanalysis of randomized, controlled clinical trials. J. Hypertens. 23, 475-481.

Witwer R.S., 2008. Natural resistant starch in glycemic management: from physiological mechanisms to consumer communications. In: Nutraceuticals, glycemic health and Type 2 diabetes. Eds V.K. Pasupuleti, J.W. Anderson. Blackwell Publ. Profes. Ames, Iowa, 401-438.

Wong J.M., Jenkins D.J., 2007. Carbohydrate digestibility and metabolic effects. J. Nutr. Rev. 137, 2539-2546.

Wu A.H., Tseng C.C., Hankin J., Bernstein L., 2007. Fiber intake and risk of adenocarcinomas of the esophagus and stomach. Cancer Causes Contr. 18, 713-722.

USDA. 2005. US Department of Agriculture. US Department of Health and Human Services. Dietary Guidelines for Americans. USDA, Washington, DC.

Accepted for print - Zaakceptowano do druku: 18.12.2013

For citation - Do cytowania

Ötles S., Ozgoz S., 2014. Health effects of dietary fiber. Acta Sci. Pol., Technol. Aliment. 13(2), 191-202. 